\title{
Global Products, Global Markets: Jeans in Korea and the United States
}

\author{
By: Marilyn DeLong, Karen LaBat, Nancy Nelson, Aeran Koh, Yangjin Kim
}

DeLong, Marilyn, LaBat, Karen, Nelson, Nancy, Koh, AeRan. (2002). Global products, global markets: Jeans in Korea and the United States. Clothing and Textiles Research Journal, 20(4), 238-245.

Made available courtesy of SAGE Publications: http://ctr.sagepub.com/

***Note: Figures may be missing from this format of the document

\begin{abstract}
:
This cross-cultural study illustrates how products selected for their basic similarities are distinguished in use by cultural context. Jeans, a product manufactured in both South Korea and the United States, were selected as the research stimuli. Subjects were regular wearers of jeans, 32 of whom were from the United States and 34 from Korea. All were females between the ages of 18 and 24, and students at one of two large metropolitan universities in their respective countries. Participants responded to four brands of jeans similar in appearance: two were U.S. brands (Levi's and Gap) and two were Korean brands (GV2 and Bang Bang). Subjects' perceptions of the products were explored for similarities and differences in criteria for wear and purchase of jeans. Country of manufacture mattered less than country of origin, appearance and other factors related to use in a cultural context. For both groups, color, fit, tactile qualities and price also were important criteria in wearing and purchasing jeans. Though respondents used similar criteria in this cross-cultural comparison, differences in weighting of those criteria yielded significantly different results.
\end{abstract}

\section{Article:}

The globalization of economic activity is affecting the flow of goods and services, creating the need for an examination of the globalization process (Hassan \& Kaynak, 1994). Understanding this process requires consideration of both product and consumer responses to the product. Because identical forms of products can be produced in many countries, a competitive edge can be developed by examining consumer's expectations of products in order to anticipate marketplace effects. Broad issues need to be addressed such as consumers' demographic characteristics and their preferences and values within a given cultural context. Investigation is needed of consumer responses to products within specific markets. In this research we study cross-cultural responses with regard to similar jeans manufactured in South Korea and the United States.

This research explores changes that occur in meaning when a product is developed in one country and adapted in another. As such, it builds upon a past study that examined responses of Korean and U.S. students to images of jeans (DeLong, Koh, Nelson, \& Ingvoldstad, 1998). Results indicated that jeans were worn for similar reasons in both countries; the reasons were comfort and versatility. However, symbolic meanings differed between the two cultures. In the U.S. jeans symbolize the culture as a whole indicating relaxed and informal attitudes that cut across all societal levels; in Korea, however, jeans symbolize youthfulness and a student lifestyle 
within the larger Korean society. In this study, we examine responses to specific jeans manufactured and worn in both countries in order to explore similarities and differences in response to the product.

\section{Products in the Global Marketplace}

Globalization can impact products in a number of ways, including the development of new products and new markets, or the adaptation or alteration of existing products. Interesting examples exist in which the purchaser changes the product form. For example, Nigerians purchased and transformed Indian madras to pelete bite cut cloth in order to better integrate the product with the culture's values and traditions (Eicher \& Erekosima, 1982). This suggests that as the form of a product is altered, a change occurs in its original meaning.

Globalization, however, also can lead to a more widespread distribution of well known products that are not altered in form. When identical forms are adopted, do their meanings change? How do people perceive and respond to similar products that are found in markets around the globe? Objectively comparing the forms of products is not sufficient as this does not address how they are perceived and how their meanings may be altered within the context of different cultures. When a product appears similar in form but is perceived differently by people within two distinct cultures, such nuances in meaning often can pose marketing challenges.

\section{Jeans in the Global Context}

Jeans originated in the U.S. where they have had a long history of manufacture and use. By the second half of the twentieth century, consumers in other countries had adopted them. However, in the process they may have taken on a global identity unique in comparison to other products. As a result, the meaning of jeans may not be tied to any one culture (Kaiser, 1997; AbrahamMurali \& Littrell, 1995; Roach-Higgins, Eicher \& Johnson, 1995; Damhorst, Miller \& Michelman, 1999). If this is so, then the question to be asked is, do global markets create global products with cross- cultural meaning or do products in the global market take on different meanings based on the country of distribution?

Some countries, such as South Korea, not only are importing jeans, but manufacturing them as well, and applying their own brand names. Country of manufacture, then, may become an issue of adoption when consumers have the choice of selecting goods produced in their own country versus those produced elsewhere.

Knowledge of cultural context is critical for designers and manufacturers in maintaining market advantage. Well- recognized brand names that have been marketed globally, such as Levi's, are facing fierce competition from private label jeans, and as a result are experiencing externally imposed change such as layoffs and downsizing. Understanding market uniqueness is a necessity if market share is not to diminish (Dickerson, 1991; King \& Bounds, 1997). Recognition of the diversity of product markets is often crucial to the success of a product, and altering a product in response to cultural context may improve a company's competitive advantage.

Nesbitt (1997) viewed Asian markets as a potential consumer gold mine. Although Asian countries are currently facing economic difficulties, the Korean economy is predicted to remain dynamic (SaKong, 1984). Economists warn of the dangers of perceiving Asians as one group, 
and marketing too broadly to a general Asian market. Each Asian country is an autonomous society with a unique culture and therefore its consumers have different needs and expectations (Kalman, 1997). Each culture has its own idiosyncratic values relevant to the use of a particular product such as jeans, and may be marketing domestic brands of jeans that are suited to the needs and expectations of local consumers.

An examination of advertisements in Korea of U.S. branded jeans indicates that they are marketed using most of the same text and visual information as is used in the U.S. Thus marketers are not acknowledging the uniqueness of the Korean market and seem to assume that the Korean market is identical to that of the U.S.

\section{Response to Global Products}

International product marketing should involve understanding responses of people to products within given cultural contexts. Such responses include aesthetic evaluations. Aesthetic response is the study of ways people experience materials of culture through selection, use, and valuethat is, an individual or collective conception of what is desirable (DeLong, 1998). For apparel products, aesthetic response involves understanding how knowledge and expectations of and experience with design influence a particular individual or cultural group. This includes examination of a product's form and meaning within a cultural context.

Apparel has meaning within a cultural context. Values can range from those that are meaningful to a given individual, to those that are shared cultural norms (Lachmann, 1991, p. 308).

Rubenstein (1995) writes that clothing symbols "...reflect ideas about what is valued in a society at a particular time," and that such meanings may be multiple as a result of the interpretation of those values from various reference points (p. 11). Rubenstein argues that the individual wearer cannot be easily understood through clothing symbols because physical objects can take on numerous values and those values are manifested in different ways (e.g., the relationship between the wearer and the object is of importance to the understanding of meaning).

Holman and Weiner (1985) used jeans to explore how the values and lifestyle (VALS) typology can provide insight into the connection between products and people. The authors suggested a relationship between VALS and the purchase of different brands of jeans. That is, participants discriminated among brands of jeans in three ways: product attributes, benefits, and values related to images.

In the case of designer-label jeans, while the basic style may be typical of jeans, the label serves as a symbol for the wearer to communicate both social and economic messages to others (Morgado, 1993), which are then understood through an established value system. Feinberg, Mataro and Burroughs (1992) explored the relationship between the meaning of jeans and the personality of the wearer using five drawings of jeans that were identical except for the brand name on the back pocket. Participants were able to differentiate social information present in the brand labels, but selection did not vary by the wearer's personality.

Davis (1989) sees the culturally encoded meaning of brand-name jeans as a paradox: on the one hand, a reflection of the desire for individual distinction while on the other hand, symbolic of a need to adhere to an ethic of asceticism within Western cultures. Workman (1988) examined 
symbolic quality related to types of jeans. Inferences made about an individual were based on choice of a particular type of jeans. Participants who were primarily female were presented with a shopping list for clothing that differed only by brand or type of jeans. They reported a more socially desirable image for designer jeans at the time of the study. Yet products can convey many images. As McCracken (1989) writes:

The social sciences have for too long imagined that status meaning is the chief meaning carried by the material culture of a consumer society. . . It is time to see that goods carry many meanings additional to those of status, and that some of thesemeanings are very deliberately at odds with the status system and the objectives of conspicuousconsumption. (p. 177)

Holbrook (1994) argues for an axiology of clothing wherein the ". . value resides not in the product purchased but rather in the consumption experience derived there from" (p. 134). He defines customer value as ". . . an interactive relativistic preference experience" and posits that all issues are in relation to one another in respect to consumer value and the purchase of products (p. 132). Jeans have extrinsic as well as intrinsic value - in that they are considered to fulfill a purpose outside of the product itself - that of durability, longevity of wear and utilitarian function. But jeans are admired and worn for their intrinsic value-flattering fit, right color, as part of the whole of the ensemble that a person enjoys wearing, providing positive aesthetic experiences.

\section{The Origin and Use of Jeans}

Jeans were chosen as the focus product in this study because they are widely available and fairly consistent in product features. While fashion trends affect the overall silhouette of jeans, basic construction and physical character tend to remain similar across time. They are typically fabricated of denim, and dyed indigo blue. They generally include five pockets, a fly-front, and topstitching along the pockets and inseam.

Jeans were first manufactured in the U.S. for nineteenth- century miners, and have continued with a rich and varied history (Hall, 1992). Throughout the twentieth century, jeans were a consistent product in wardrobes of U.S. males, but in the early decades typically were worn while performing physical labor rather than as a fashion statement. Today jeans commonly are worn by both men and women of all ages for a variety of activities. For example the Baby GapTM label offers a selection of jeans for toddlers, and it is certainly not uncommon to see jeans worn by older individuals in the U.S.

The history of jeans in Korea is much shorter than it is in the U.S. Jeans were first introduced in Korea through black market trading by American soldiers after the Korean War. In the 1960s, jeans, acoustic guitars and draft beer became symbols of the youth culture, and in the 1970s jeans were popular among university students who were accustomed to wearing uniforms in their early school years. Also, jeans represented resistance to older generations because jeans were once prohibited. When the Korean government opened the market in the late 1980s to include more imports, U.S. branded jeans became more widely available than before.

Today Korea manufactures a range of national brand and designer jeans. Koreans tend to choose brand names that sound like U.S. brand names and expect to pay high prices for designer and specialty brands. The primary wearers of jeans are young people, especially students. In fact, in 
Korea, wearing jeans is considered a badge of the student (DeLong, et al., 1998). Koreans are generally more formal in their dress than the casual nature of jeans allows, and Koreans of all age groups typically do not wear jeans.

It is important to note that many of the studies reported in the literature focus on Western paradigms in which meaning and value are implicitly understood. Most do not address the possibility of studying products in countries that hold different values. Morris and Hallaq (1994) evaluated Korean perceptions of U.S. and Japanese products with those made in their own country. Their study indicates that though Koreans may regard their own brand-name products as inferior to those of other countries, they still prefer domestic Korean products to those of other countries, with U.S. branded products second, and Japanese third.

Korea has a history of manufacturing products designed elsewhere. For example, the U.S. often has designed products that are manufactured in Korea. Thus in Korea, country of manufacture may not provide impetus to purchase as much as brand loyalty, which offers a set of expectations based upon product familiarity. Brand loyalty is a factor that may lessen the influence of country of manufacture, especially with a consistent product such as jeans. "[B]rand loyalty is always conditional" and subject to change (O'Shaughnessy, 1987, p. 40). Therefore both country of manufacture and brand name are product characteristics explored in this study.

\section{Methods}

\section{Subjects}

University students in both Korea and the U.S. served as subjects in this study. Subjects were recruited from two large universities; one in Korea and one in the U.S. Flyers were posted throughout the universities to solicit subjects. All subjects were women, aged 18 to 25, and regular purchasers and wearers of new jeans. If potential subjects met the criteria of age and regular wear of new jeans, appointments were made for groups of four to ten to facilitate individual and group questioning procedures. Thirty-four students participated in Korea and 32 students in the U.S.

\section{Selection of Stimuli}

Four pairs of jeans, two U. S. brands and two Korean brands, were selected as stimuli for the study. The Korean jeans were chosen first and sent to the U.S. where they were taken into stores and compared to find similar U.S. jeans. The Korean jeans were one national brand and one specialty brand. National brand is defined as a jean designed and produced within the country and sold through many retail outlets. A specialty brand jean is designed and contracted for manufacture by a retailer and manufactured within the country. Criteria used to select the U.S. jeans were to select

a national brand and a specialty brand and to match as closely as possible color, detailing, size, and shape. A U.S. Levi's branded jean was selected as similar to a Korean jean labeled Bang Bang. These labels represent national brands in both countries. Gap (U.S.) and GV2 (Korea) were selected because they are examples of specialty brands within each country. Gap jeans are sold by a specialty retail store widely recognized in the U.S., while GV2 is one of the specialty brands in Korea and reported as a highly popular brand in a 1996 market study (Texjournal, 1996). All jeans were size 6, medium value indigo blue denim, 5-pocket, gold to brown topstitching, with slim fit silhouette. 
The price and market availability of the jeans differed between the two countries. Neither Korean brand was available in the U.S, so it was unlikely that U.S. subjects had seen these brands. Levi's is available in Korea, but prices are higher than in the U.S. with U.S. Levi's priced at $\$ 35$ to $\$ 40$ and Korean imported Levi's at $\$ 75$ to $\$ 95$. The Korean national brand (Bang Bang) was priced at $\$ 43$. Both specialty store brands were higher in price with U.S. Gap jeans costing $\$ 40$ to $\$ 60$ and the Korea GV2 costing the equivalent of $\$ 140$. Prices of jeans were not revealed to subjects at the time of the study, but subjects may have been familiar with costs.

\section{Questionnaire Development}

Three questionnaires were developed to assess subjects' preferences for the four jean types. A bilingual Korean researcher translated the questionnaires into Korean for the Korean subjects.

Questionnaire \#1 was used to assess subjects' evaluation of overall appearance and quality of the stimuli jeans including stitches, fabrics, and features such as color and size of buttons and rivets. Responses were recorded using a 10-point bipolar scale, where 10 meant "Excellent" and 1 meant "Poor." Additional questions assessed subjects' preferences for the product asking, "How much do you like this product?" "How much do you think you would like to wear this product?" and "If you were currently planning to purchase such a product, how likely would you be to purchase this one?" Additionally, some questions were open-ended asking "why" or "why not?"

In Questionnaire \#2 subjects were asked to list their reasons for wearing jeans and to rank the four jeans according to overall preference. Subjects were also asked to indicate which jeans, of the four, they would most likely purchase. Then subjects were asked how many times per week they wore jeans, to describe when they wore them (on what days of the week, and for what occasions), to describe their favorite pair of jeans in detail, and to list the attributes that they find most important about their favorite pair of jeans.

Questionnaire \#3 consisted of a set of prompts used by the leader of focus group sessions. These questions were formulated to give subjects the opportunity to further explain their responses in questionnaires \#1 and \#2. The following questions were asked:

- What is the most important feature of a garment thatprompts you to buy it? For jeans?

- Do you expect different features from products madein different countries? What about jeans specifically?

- Why do you wear jeans? The remainder of the questions listed below included theprobe, "why or why not"

- Does the brand name affect your decision to purchase?

- Does the country of manufacture affect your decision to purchase?

- Consider this comparison of two pairs of jeans — one is a U.S. brand and the other Korean. Would you purchase either one?

- When considering what to wear with jeans, do you consider jeans as 'going with' any ensemble or do you wear only certain items with jeans?

- Do you believe jeans are a symbol of U.S., Korean, or world culture?

\section{Procedure}

Procedures followed were identical in both countries and all data were collected within a period of one week in the same month. Subjects were ushered into a research room where they completed all steps of the research. The in-depth nature of the research meant each participant spent between one and one-half to two hours to complete the research protocol. Subjects first 
completed human subjects permission forms. Then subjects completed questionnaires \#1 and \#2. On completion of the questionnaires, a facilitator using a focus group process using question set \#3 questioned groups of four to six subjects. This session was audio taped with the subjects' permission (included in the consent form), and was then transcribed by the researchers.

As part of Questionnaire \#1, subjects were asked to examine and evaluate the four stimuli jeans. Jeans were placed at four separate stations. Labels were left on the jeans, as purchased, in order to cue the subjects as to country of manufacture and to provide the identifying brand label.

Subjects evaluated the jeans individually so that only one subject was allowed at each station at any given time.

Responses were analyzed using frequencies and analysis of variance. Responses to open-ended questions were coded and analyzed using cross tabulation between the two groups as well as between the two countries.

\section{Results}

\section{Questionnaire Results}

Responses were compared for each of the products. Table 1 presents the mean responses of the subjects in each country. Responses were significant at the .01 or .001 level for the following: overall quality and appearance of the product, how much subjects liked the product, how much they would like to wear them, and how likely they would be to purchase them.

Responses to the products between the two countries suggested a difference in perceptions of the jeans. In the U.S. the ratings were higher for the two U.S. brands, Levi's and Gap, and respondents indicated they were most likely to purchase these brands. In Korea the means of the product ratings were higher for GV2 and Gap, a Korean and U.S. brand respectively.

Korean subjects favored the two specialty brands, Gap

Table 1. Mean Responses of U.S. and Korean Subjects in Evaluating Stimuli Jeans (Questionnaire \#1)*

\begin{tabular}{|c|c|c|c|c|c|c|}
\hline Country & Variable & Levi's & Gap & GV2 & Bang-Bang & $F$-value \\
\hline \multirow[t]{5}{*}{ USA } & Overall appearance & 7.59 & 7.44 & 6.53 & 5.84 & $11.75 * * *$ \\
\hline & Overall quality & 8.00 & 7.28 & 6.47 & 5.94 & $3.58 * * *$ \\
\hline & How much do you like this product? & 7.38 & 7.66 & 5.81 & 4.84 & $20.63 * * *$ \\
\hline & How much do you think you would like to wear this product? & 7.22 & 7.68 & 5.63 & 4.66 & $15.19 * * *$ \\
\hline & How likely would you be to purchase this product? & 7.06 & 7.06 & 4.81 & 3.97 & $14.54 * * *$ \\
\hline \multirow[t]{5}{*}{ Korea } & Overall appearance & 4.53 & 6.53 & 6.77 & 5.21 & $17.31 * * *$ \\
\hline & Overall quality & 6.56 & 6.56 & 6.94 & 5.82 & $4.21 * *$ \\
\hline & How much do you like this product? & 4.71 & 6.03 & 6.85 & 4.21 & $17.32 * * *$ \\
\hline & How much do you think you would like to wear this product? & 3.50 & 6.12 & 6.74 & 3.24 & $28.50 * * *$ \\
\hline & How likely would you be to purchase this product? & 2.91 & 5.47 & 5.56 & 2.71 & $18.94 * * *$ \\
\hline
\end{tabular}

\footnotetext{
*Based on a 10 point scale; $10=$ high and $1=$ low

$* * p<.01$

$* * * p<.001$
} 
Table 2. Frequencies Given by U.S. and Korean Subjects on Criteria for Evaluating Jeans (Questionnaire \#2)

\begin{tabular}{lrr}
\hline Criteria & Korea & U.S. \\
\hline Brand Name & 16 & 8 \\
Color & 29 & 22 \\
Comfort & 2 & 6 \\
Details & 10 & 8 \\
Fabric Quality & 3 & 6 \\
Fit & 1 & 16 \\
Price & 4 & 10 \\
Fit of rise & 15 & 5 \\
Style/Design & 18 & 8 \\
Tactile Qualities & 11 & 10
\end{tabular}

and GV2, for all five of the variables. GV2 was preferred but Gap compared very favorably (Table 1). Subjects evaluated the quality of the four products similarly (Table 1). However, the responses yielded differences at $\mathrm{p}<.001$ for all four products on overall appearance, how much they liked and would wear the product, and how likely they would be to purchase the product (Table 1). The widespread differences in Korean responses among the brands indicate that specialty labels were consistently favored. Thus brand name was favored over country of manufacture.

\section{Results of Product Examinations}

Table 2 shows the frequency of the top five criteria participants listed for wearing jeans. For U.S. subjects, color was ranked the most important criterion for wearing jeans, fit was ranked second, both the tactile nature of denim and price were tied for third, style/design details and brand name were tied for fourth, and fabric quality and comfort were ranked fifth. Similarly, Koreans ranked color number one. Second, however, were factors of appearance such as style and design. Brand name was ranked third as a criterion for wearing jeans. However, there was much more agreement by the Korean subjects as compared to the US subjects, suggesting that brand name is possibly more important to the

Table 3. Frequencies of U.S. and Korean Subjects on Which Stimuli Jeans They Would Purchase

\begin{tabular}{lcccccc}
\hline Country & Levi's & Gap & $\begin{array}{c}\text { Gap or } \\
\text { Levi's }\end{array}$ & GV2 & $\begin{array}{c}\text { Gap or } \\
\text { GV2 }\end{array}$ & $\begin{array}{c}\text { Bang } \\
\text { Bang }\end{array}$ \\
\hline US & 8 & 11 & 7 & 2 & 1 & 2 \\
Korea & 1 & 14 & 0 & 13 & 2 & 3
\end{tabular}

Korean subjects. Concern with fit of the rise was the fourth criteria for Korean subjects. Tactile qualities of the fabric ranked fifth. These data support and correspond with focus group responses in both countries.

Based on the criteria for wearing jeans they had previously listed and ranked, necessitating brand recall, subjects were asked to respond to which of the four jeans they would purchase. Jeans that subjects said they would most likely purchase are shown in Table 3. Koreans chose Gap as their first choice, GV2 second, Bang Bang third, and Levi's last behind the response Gap or GV2. Similarly, U.S. subjects ranked Gap number one, Levi's second, with Gap or Levi's third, GV2 
and Bang Bang tied for fourth, and Gap or GV2 ranked last. Responses in Tables 1 and 3 differ, possibly indicating that the responses shown in Table 3 are based on what the subjects had seen.

\section{Results of Focus Group Discussion}

An interpretation of the data that combines questionnaire responses with the focus group responses gives insight into cultural differences. Both the U.S. and Korean groups were familiar with jeans as a popular form of dress; however they differed in the extent of wearing. U.S subjects cited wearing jeans more often at five to seven times per week than Koreans, who cited three to five times per week. Color was an essential factor in the appearance of jeans for both U.S. and Korean subjects. In focus groups many stated that they would not be interested in trying on jeans that were of a color they disliked, a decision they made immediately upon seeing the jeans. The importance of color was apparent to both groups of subjects, since they indicated that jeans can be worn with anything, provided they are the "right color." U.S. subjects went so far as to point out that they have jeans in various shades of blue for different occasions depending on the degree of formality/informality of the event - dark blue being appropriate for more formal situations, and light blue for casual times. The preferred color was light blue for everyday wear for subjects in both countries.

The cut of jeans was also an important criterion of appearance. In focus groups, Korean subjects indicated that U.S. national brand jeans were not made to fit Korean body proportions, tended to be longer in the waist and legs, and baggier in the thigh area than Korean jeans. U.S. responses indicated the importance of finding just the right proportion of length and width. Finding an inseam length to correspond with leg length was a concern for almost all of the U.S. subjects. Many mentioned choosing men's jeans because of more specific size options. For both Korean and U.S. subjects, "good fitting" jeans should not be too baggy or too tight in the waist, hips, and thigh areas.

During the focus group discussions, U.S. subjects were asked if they would wear Korean brand jeans. Subjects were generally concerned with how Korean brand jeans would fit, and particularly that the inseam would be shorter and the proportions smaller than those of U.S. jeans. Likewise, Koreans felt the same way about Levi's, a U.S. brand, in that they were not made to fit the Asian figure, and suggested that this affected the design of the jeans. This is most likely the reason for their low ranking in terms of Korean subjects' intent to purchase jeans. It is important to note that U.S. perceptions of Korean jeans as being ill-fitting is not based on prior experience or exposure to the brands, while in contrast, the Korean response to the U.S. national brand (Levi's) could have been based on past experience with their fit. In both instances, groups were responding to actual U.S. and Korean brand jeans that were as similar in size and cut as possible. Thus, their responses in both cases were more likely based on past experience with fit than on the jeans used as stimuli.

According to U.S. and Korean focus group responses, jeans can be worn with anything, provided they are the right color and fit. U.S. responses indicate that the specific cut of jeans (i.e., loose or slim fit) looks better with certain kinds of tops. U.S. participants responded more often than Koreans that while jeans may go with most ensembles, there are particular ways in which tops are coordinated with jeans. Koreans responded in a more general sense, replying that jeans can be worn with anything unless it is a "formal" or dressy situation. 


\section{Discussion}

An interpretation of these data is that U.S. participants responded based upon country of manufacture and Koreans responded based upon appearance and brand expectations. For Koreans, prestige of the brand was important. However, product familiarity is also a possible interpretation, and this reasoning was cited in the focus group sessions. The Korean brands, Bang Bang and GV2, were unknown brands to U.S. subjects. While all of the brands were available in Korea, the Gap brand was less familiar than the others.

Tactile qualities were important to participants in both countries. Such qualities include the feel of the fabric, in terms of softness or stiffness, and issues related to physical comfort on the body. Softness was a desired tactile quality for participants in both countries, and likely tied to color. That is, light blue colored denim indicates that the jeans have been through a bleaching or distressing process that usually softens the fabric. In addition, U.S. participants were more concerned with the quality of the denim fabric than Koreans, and ranked fabric quality in terms of durability and wear more highly than other criteria. Such qualities can be determined by handling the jeans in the store before purchase. Fraying at the hem and lack of weave density can indicate poor quality denim. Decisions having to do with quality of fabric are likely to be based on past experiences with the durability and wear of brands of jeans.

Subjects in both countries indicated that their interest was limited to jeans they could afford to purchase. Some U.S. subjects stated in the focus groups that they would not try on a new brand of jeans that was too expensive, out of concern that the jeans would fit beautifully and they would feel compelled to purchase them. This may be why U.S. subjects ranked price of product as an important criterion, far more important than indicated in the Korean ranking. However, the most preferred jeans brand for U.S. participants was Gap, also the most expensive U.S. brand of the four pairs.

Korean and U.S. subjects responded differently about design and specific design indicators such as labels, signature markings or other details such as stitching design and placement. Koreans believed Korean jeans exhibited good design and they liked visible indicators of brand name on them. In contrast, U. S. respondents wanted little or no visible indicator of brand. Details such as color of stitching and rivets appeared to be more important to U.S. participants.

Brand was important because it suggested familiarity. For both groups the primary concern with brand was prior knowledge and perception of fit. In both the U.S. and Korea, the symbolic properties of jeans, regardless of brand, were related to notions of who wore jeans in each country. However, responses indicated that the status of brand names is more important for Koreans than for U.S. consumers. When Koreans see select brand names, they assume both quality and prestige. The responses of U.S. subjects may be explained by Holbrook's (1994) notion of the "interactive relativistic preference experience," wherein the individual is at the center of the experience as jeans consumer. For Korean students family status is considered uppermost and the older generation is influential with the young. Thus, appearance is extremely important for young Koreans, and in particular, wearing recognized brand names. 
Country of manufacture was not as important to the participants as were overall appearance and fit. Students indicated that country of manufacture normally did not affect their decision to buy, but familiarity that set up expectations did. U.S. subjects were not familiar with either of the Korean brands, GV2 or Bang Bang, and therefore did not score them as high as the two U.S. brands. Both Korean and U.S. subjects scored Bang Bang as their last choice. However, Koreans, who were familiar with the Bang Bang brand indicated they were far less likely to purchase them than U.S. subjects. The difference could be the result of the Korean subjects having a negative experience when purchasing and/or wearing this brand. But this could implicate some brand name equivalency between the two countries in that the difference in preference between the two U.S. jeans was less than that between the two Korean brands.

Reliability of the products based on past experiences was important for both groups. This reliability was expressed in several ways; knowledge that the jeans will fit, that they wear well, or that the cut and color of the jeans go with most anything. Some Koreans mentioned that specialty brand names, Gap and GV2, typically meant better quality.

Expectation based on familiarity and prior satisfaction/ dissatisfaction was another criterion. Brand loyalty may not be as much about indexing social or economic status as it is about prior knowledge of satisfaction or dissatisfaction with past purchases and use of the product in general. A degree of loyalty may develop to the extent that the consumer assumes that the product will meet criteria based upon expectations. It was also possible that the perception of the jeans was based on prior experiences unrelated to this specific product. For example, U.S. participants expressed concern about fit of the Korean jeans given the difference in standardized body measurements between the two countries, as did Koreans about U.S. brand jeans. Thus a degree of loyalty develops, to the extent that the consumer assumes that the branded product will meet the necessary criteria.

In discussing values and expectations participants addressed the form of the product; however, cultural context provided an interpretation of meaning that helped shape the responses of subjects in both countries. For example, in the focus groups, U.S. subjects responded that jeans symbolized youth, comfort, and an "American" attitude. In contrast, Korean participants responded that jeans symbolized world culture in general, but youth culture in their own country. While Korean subjects acknowledged that the origins of jeans wearing took place in the U.S., they responded that jeans are now being worn all over the world and, as a result, have taken on broader and more global meanings.

\section{Conclusions and Implications for Further Research}

This study highlights the necessity of focusing on responses to real clothing products to discover nuances of importance given to product selection criteria within a cultural context. Four jeans were selected that appeared as similar as possible, two manufactured in Korea and two in the U.S. Evidence from examining the responses of female participants of the same age in the two countries showed that jeans were perceived differently, although the subject's criteria for evaluation were similar. While the process of evaluating jeans was similar in both countries, the importance of the different criteria, and the outcome of the evaluations were markedly different. 
Differences in brand preference between the U.S. and Korean responses were found. While the Korean subjects indicated highest preference for the U.S. specialty brand jeans, the appearance of the product rather than past experience with brand name or country of manufacture influenced that preference more. Though country of manufacture was on the label, Gap jeans were not widely known or available in Korea at the time of the study. Further, focus group data indicated that the issue of country of manufacture seemed of little importance to either U.S. or Korean subjects when thinking about purchase. This may be due to a past history of extensive interchange of products between the two countries, with Korea being a primary and long time manufacturer of U.S. products.

The role of culture with respect to global products becomes clear upon examination of the response criteria of subjects from the two countries. In both countries, comfort was a reason given for wearing jeans. While factors such as fit are important in the two cultures, the degree of importance of fit differed as well as concerns with kinds of fit. Another example is the criterion of brand name, in that it was listed for both countries but was more important for Korean than U.S. subjects. Both examples are clear indications of how a product can cross cultural boundaries while also serving to maintain culture-specific factors of meaning and identity. The cultural context of product use is a crucial factor for interpretation of values and expectations as part of a person's response to a global product.

The use of different kinds of data gathered from individual responses and group discussion provided in-depth understanding, although it was also a limitation of the study. Time commitment required of subjects limited numbers of volunteers and their willingness to participate. Future studies might include men's perceptions and preferences for jeans. Comparisons could be made between men and women in order to explore issues of gender identity and sexuality as communicated through jeans.

Cross-cultural communication between researchers was necessary, in order to best determine which jeans products to use in the study, as well as to gain an in-depth understanding of responses in both cultures. This relationship helped in the interpretation of the data to determine what was unique about each market.

It is clear that there is need for designers, manufacturers, and retailers to understand response criteria to products in the culture where the product will be marketed. Jeans are an ideal product to study because of their widespread use and visibility throughout the world providing the potential of soliciting an array of meanings within a cultural context. As products become increasingly global in use, it is imperative to study and understand similarities and differences of consumers' preferences and perceptions.

\section{References}

Abraham-Murali, L., \& Littrell, M. (1995). Consumers' conceptualization of clothing attributes. Clothing and Textiles Research Journal. 13(2), 65-74.

Damhorst, M.L., Miller, K., \& Michelman, S. (1999). The meanings of dress. New York: Fairchild.

Davis, F. (1989). Of maids' uniforms and blue jeans: The drama of status ambivalences in clothing and fashion. Qualitative Sociology, 12(4), 337-355. 
DeLong, M. (1998). The way we look (2nd ed.). New York: Fairchild.

DeLong, M., Koh, A., Nelson, N., \& Ingvoldstad, A. (1998). Jeans: A comparison of perceptions of meaning in Korea and the U.S. Clothing and Textiles Research Journal, 16(3), 116-125.

Dickerson, K. (1991). Textiles and clothing in the international economy. New York: MacMillan.

Eicher, J. \& Erekosima, T. (1982). Pelete Bite: Kalabari cut-thread cloth. St. Paul, MN: Goldstein Gallery, University of Minnesota.

Feinberg, R. A., Matero, L., \& Burroughs, W. J. (1992). Clothing and social identity. Clothing and Textiles Research Journal, 11(1), 18-23.

Hall, L. (1992). Common threads, a parade of American clothing. Boston: Little, Brown \& Company.

Hassan, S., \& Kaynak, E. (Eds.). (1994). Globalization of consumer markets. New York: International Business Press.

Holbrook, M. B. (1994). Axiology, aesthetics, and clothing: Some reflections on the old school tie. In M. R. DeLong, \& A. M. Fiore (Eds.), Aesthetics of textiles and clothing: Advancing multi-disciplinary perspectives, ITAA Special Publication \#7, pp. 131-41. Monument, CO: International Textile and Clothing Association, Inc.

Holman, R. H., \& Wiener, S. E. (1985). Fashionability in clothing: A values and life-style perspective. In M.R. Solomon (Ed.), The psychology of fashion (pp. 87-98). Lexington, MA: D.C. Heath and Company.

Kaiser, S. B. (1997). The social psychology of clothing: Symbolic appearances in context (2nd, revised ed.). New York: Fairchild.

Kalman, J. (1997, February). Making your goods go the distance: To Japan and Korea. Bobbin, 68-71.

King, R. T., \& Bounds, W. (1997, Tuesday, November 4). Its share shrinking, Levi Strauss lays off 6,395. Wall Street Journal, pp. B 1, B 14.

Lachmann, R. (Ed.). (1991). The encylopedic dictionary of sociology, 4th ed. Guildford, CT: Dushkin.

McCracken, G. (1989). "Homeyness:" A cultural account of one constellation of consumer goods and meanings. In E. C. Hirshman (Ed.), Interpretive consumer research, pp. 168-184. Provo, UT: Association for Consumer Research.

Morgado, M.A. (1993). Animal trademark emblems on fashion clothing: A semiotic interpretation. Clothing and Textiles Research Journal, 11(3), 31-38.

Morris L., \& Hallaq, J. (1994). The changing South Korean marketplace: Perceptions of consumer goods. In Hassan, S. and Kaynak, E. (Eds.), Globalization of consumer markets, (pp. 263-280). New York: International Business Press.

Nesbitt, J. (1997). Megatrends Asia. New York: Touchstone.

O’Shaughnessy, J. (1987). Why people buy. New York: Oxford University Press.

Roach-Higgins, M., Eicher, J., \& Johnson, K. (1995). Dress and identity. New York:

Fairchild.

Rubinstein, R. P. (1995). Dress codes: Meanings and messages in American culture. Oxford: Westview Press.

SaKong, I. (1984). An overview of the Korean economy: Past performance and future prospects. In J. K. Park, \& J. Wanandi (Eds.), Korea and Indonesia in the year 2000 (pp. 6373). Boulder. CO: Westview Press. 
Texjournal (1996). Volume 12, p. 150.

Workman, J. E. (1988). Trait inferences based on perceived ownership of designer, brand name, or store brand jeans. Clothing and Textiles Research Journal, 6(2), 23-29. 